\title{
Occurrence other than V600E mutation of BRAF gene in Papillary Thyroid Carcinoma (PTC)
}

\author{
Artur Kowalik ${ }^{1}$, Aldona Kowalska ${ }^{2}$, Janusz Kopczyński ${ }^{3}$ Agnieszka Walczyk ${ }^{2}$ Ewelina Nowak ${ }^{1}$ Elżbieta Wypiórkiewicz ${ }^{1}$, Renata Chodurska ${ }^{1}$, Liliana Pięciak ${ }^{1}$, Stanisław Góźdźz ${ }^{4-5}$ \\ ${ }^{1}$ Department of Molecular Diagnostics, ${ }^{2}$ Department of Endocrinology and Nuclear Medicine, ${ }^{3}$ Department of Surgical Pathology, ${ }^{4}$ Department of Chemotherapy, \\ Holycross Cancer Centre, Kielce, Poland \\ ${ }^{5}$ Faculty of Health Sciences, Jan Kochanowski University, Kielce, Poland
}

\section{INTRODUCTION}

$B R A F^{\mathrm{V} 600 \mathrm{E}}$ mutation constitutes $98-99 \%$ of all detected mutation in thyroid carcinoma (Figure.1). The remaining $2 \%$ are $B R A F$ mutations detected in the vicinity of codon V600. Due to the low incidence not much is known about their importance for the development of PTC. In addition, some of these mutations co-occur with the mutation $B R A F^{\mathrm{V} 600 \mathrm{E}}$. The aim of the study is to present 15 mutations other than $B R A F^{\mathrm{V} 600 \mathrm{E}}$ detected in PTC .

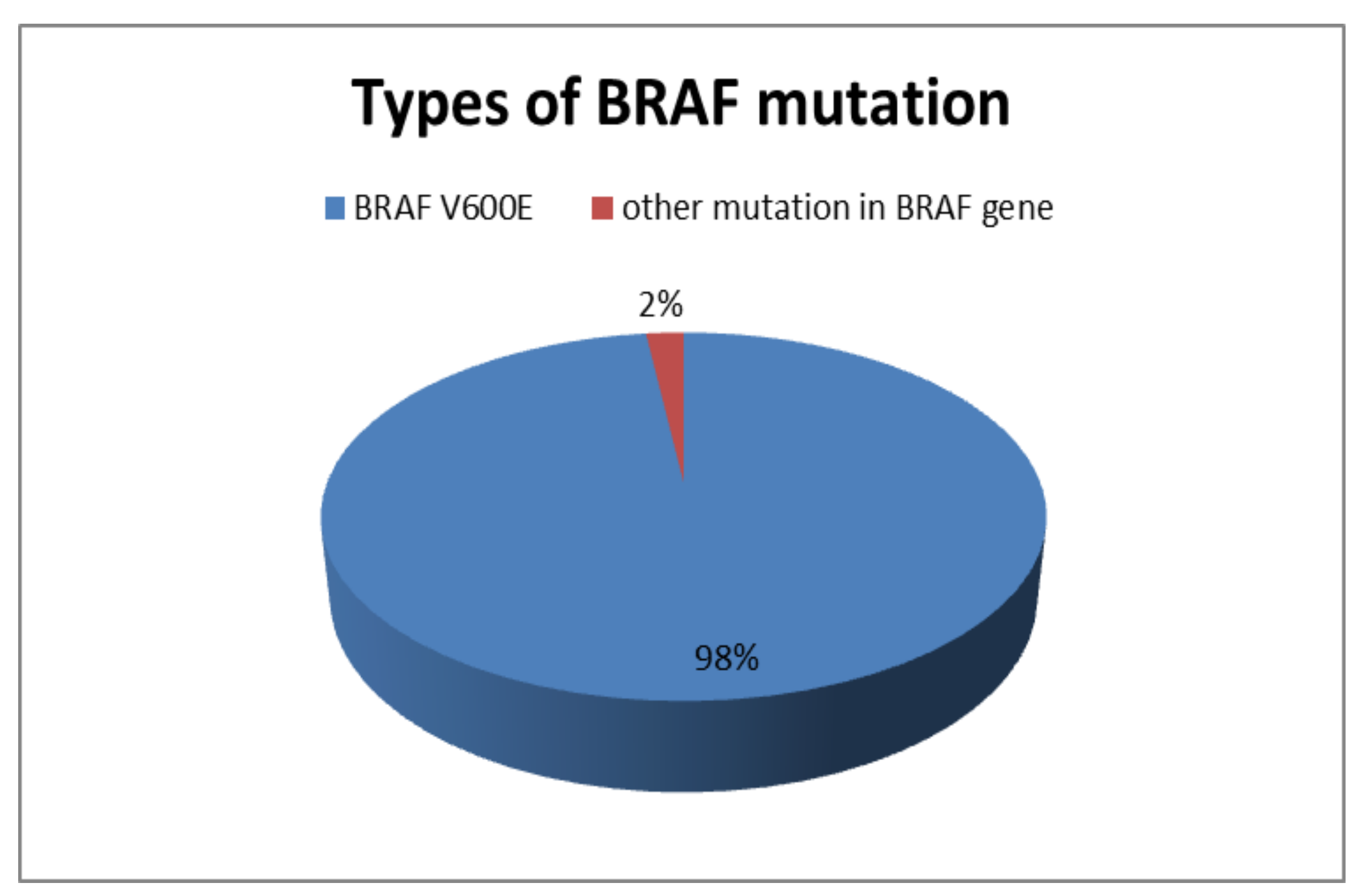

Fig. 1 Pie chart shows distribution of BRAF mutations detected in PTC

\section{MATERIALS AND METHODS}

The study presents 15 cases with mutations other than $B R A F^{\mathrm{V} 600 E}$ detected during routine diagnostics of 485 cases of PTC.

Genotyping was performed by direct sequencing. For comparative analyzes used published literature data .

\section{RESULTS}

Among the 15 mutations analyzed, three mutations (p.T599_V600insT, $2 \times$ p.V600_K601delinsE ) already detected in the PTC Fig. 2 . In contrast, mutations : p.G593S , pF610S , p.L588P , p.L584F , p.F595L , and p.D594N p.S616F already been described in cases of melanoma Fig.3 Other mutations : p.V600_604WdelinsE, p.598_599insl , p.E611K, p.S614F , p.H608Y , pG615E not detected earlier in PTC . However, in those codons previously described mutations in malignant melanoma and carcinoma of the thyroid Fig. 4. In 6 cases of PTC except V600E mutations were detected other . In one two additional ( p.G593S, pF610S ) and one in the other five cases: p.E611K, pL588P , p.V600_K600delinsE, p.H608Y, p.S616F .
A

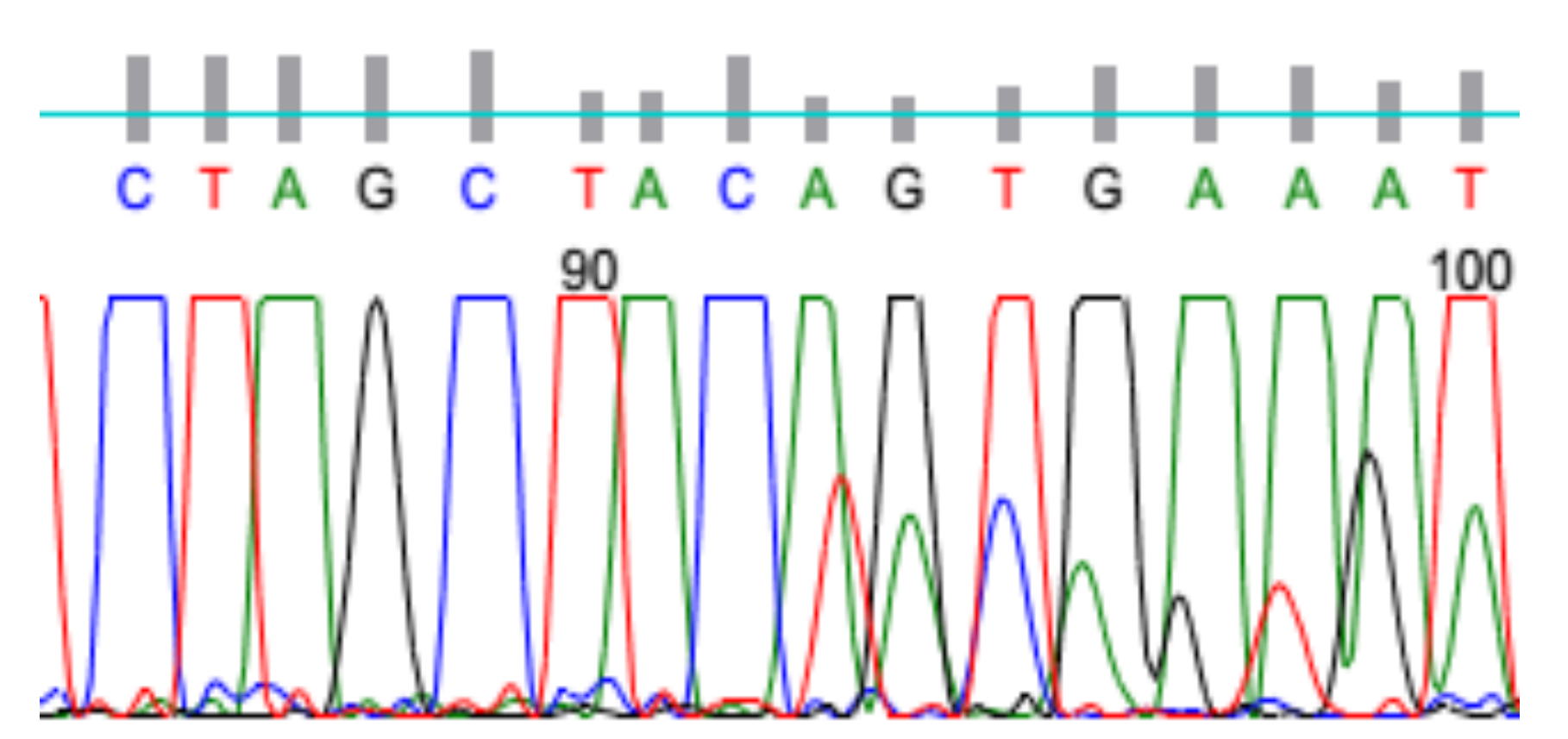

B

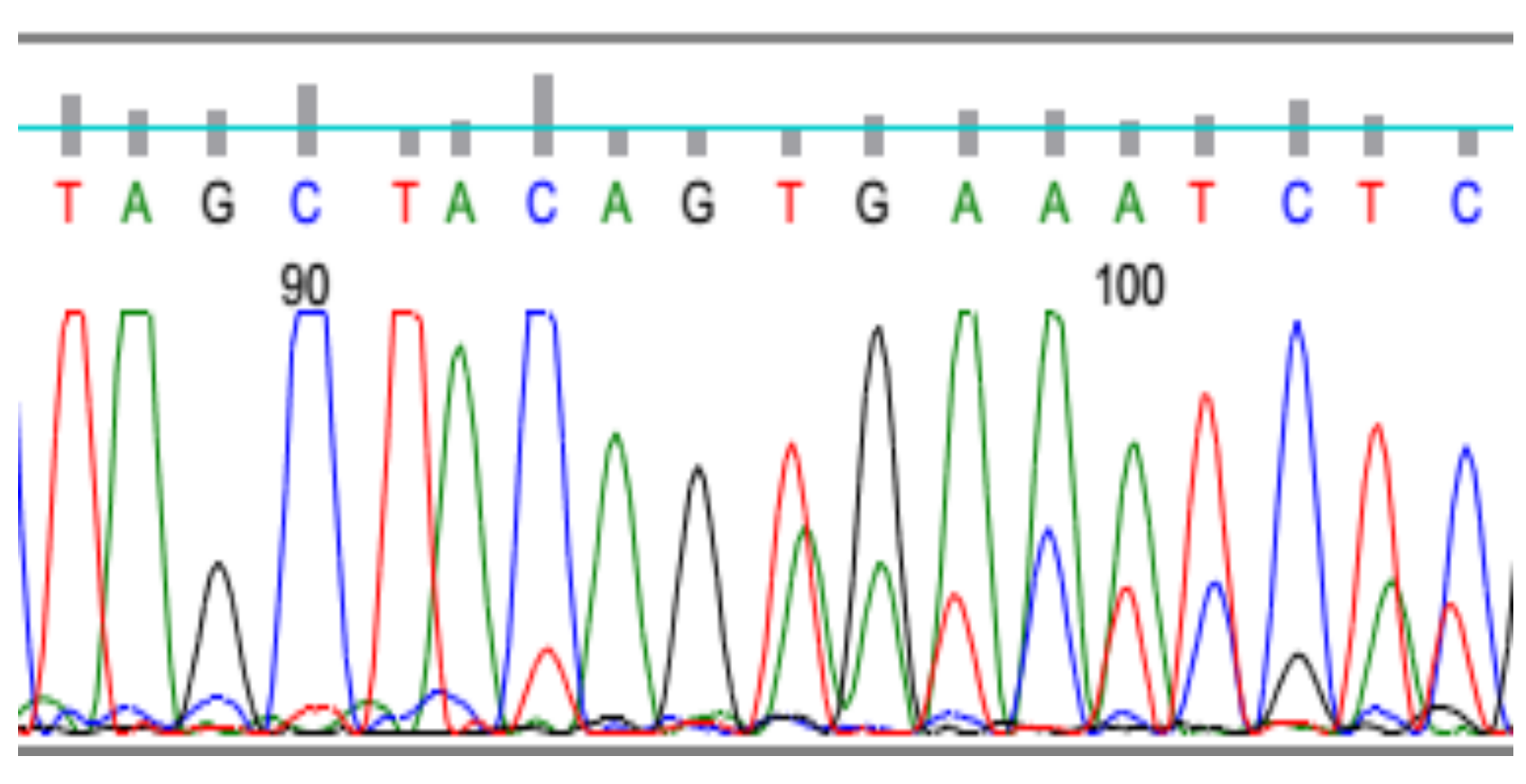

Fig.2 Representative sequencing of $B R A F$ mutations already detected in PTC. A p.T599_V600insT; B p.V600_K601delinsE;
A

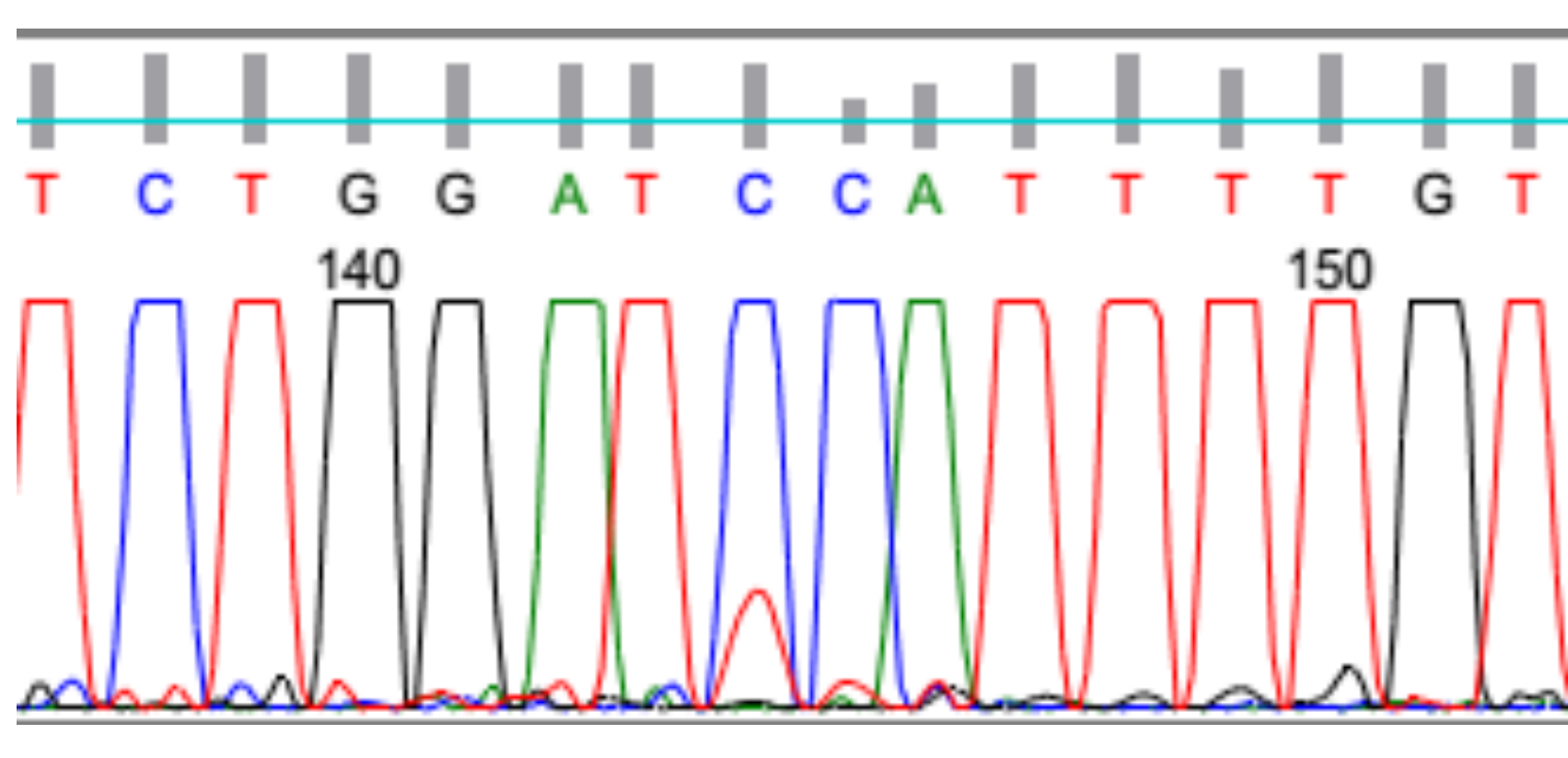

Fig.3 Examples of sequencing of $B R A F$ mutations detected in PTC and previously described in melanoma malignum. A p.S616F; B p.D594N
B

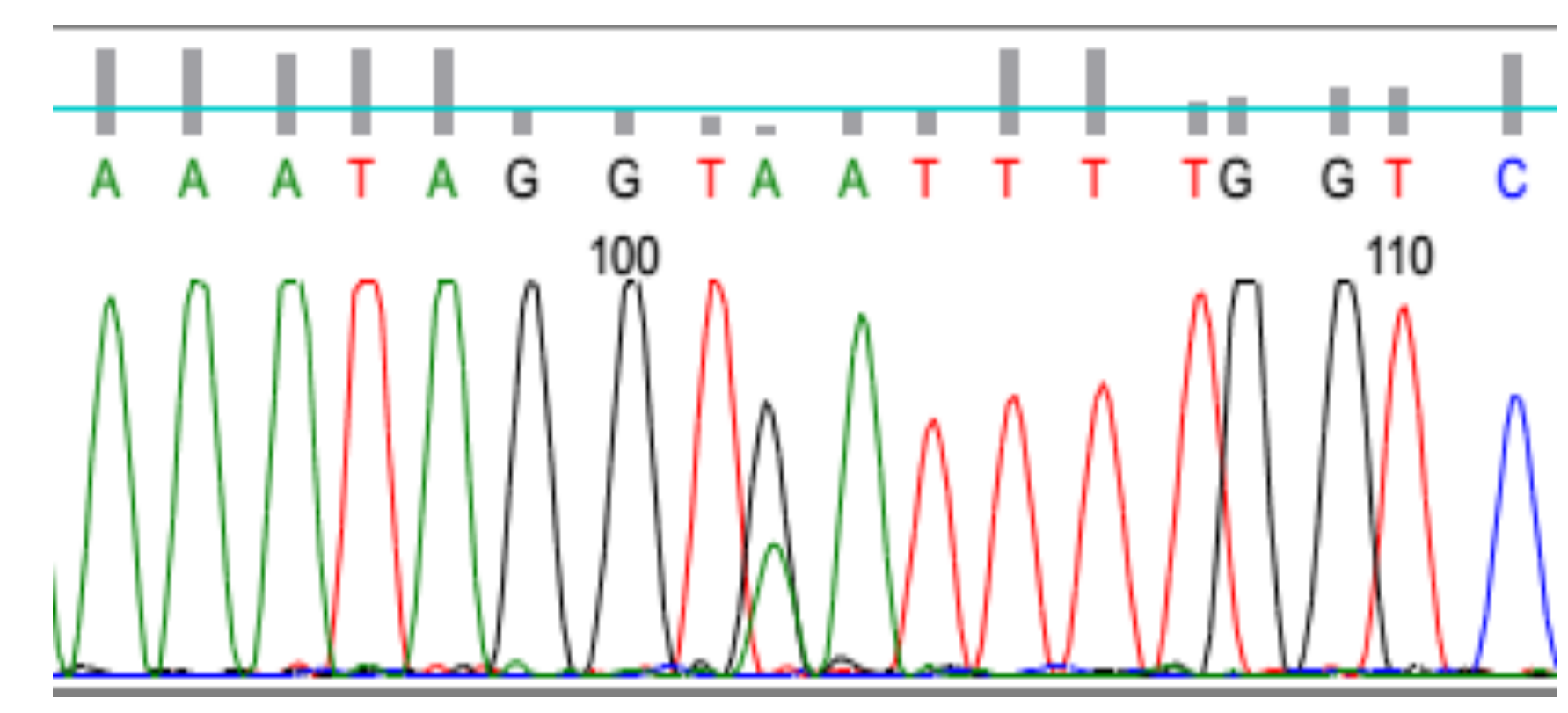

A

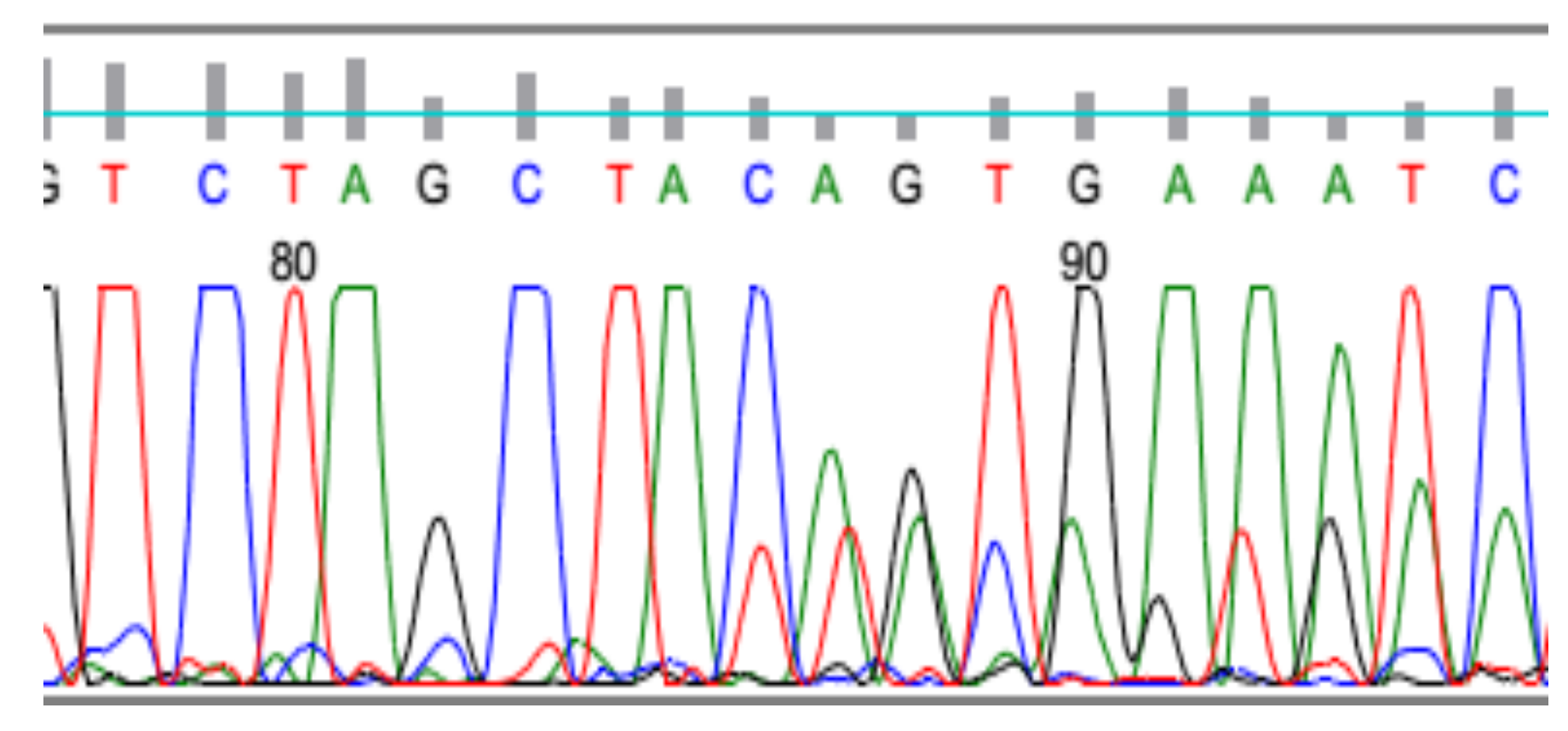

Fig.4 Examples of sequencing of BRAF mutations not detected earlier in PTC A p.598_599insl; B p.H608Y
B

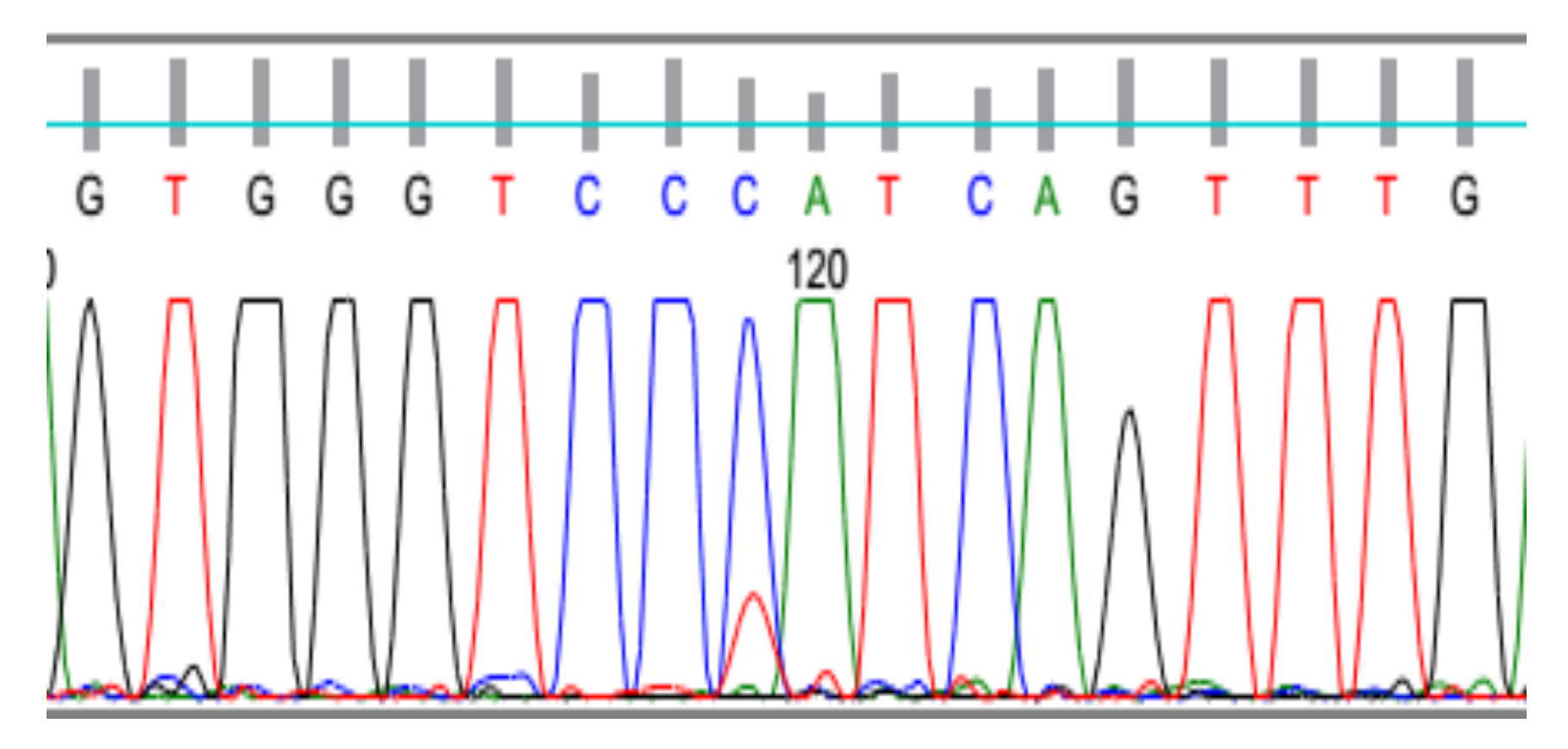

\section{CONCLUSIONS}

Mutations other than BRAFV600E because they are relatively rare in about 2-3\% of cases, and PTC mutant predominantly locate in the vicinity of the codon V600. An analysis of the literature suggests that some have already been described in the PTC and other malignant tumors (melanoma, pancreas and intestine ). This suggests that the activity of oncogenic and oncogenic potential, which may be responsible for oncogenesis $\mathrm{PCT}$. The presence of more than one mutation shows the clonality and active PTC in PTC process of tumorigenesis, which may be an important clinical outcomes . However, for a comprehensive explanation of the significance of these mutations in the oncogenesis of PTC also a need for further functional studies. 